\title{
Birth accelerations of neutron stars
}

\author{
Ricardo Heras \\ Preparatoria Abierta, SEIEM en Toluca Edo. de Mexico \\ email: ricardoherasosornor@gmail.com
}

\begin{abstract}
We suggest that neutron stars experienced at birth three related physical changes, which may originate in magneto-rotational instabilities: (i) an increase in period from the initial value $P_{0}$ to the current value $P_{s}$, implying a change of rotational energy $\Delta E_{\text {rot }}$; (ii) an exponential decay of its magnetic field from the initial value $B_{0}$ to the current surface value $B_{s}$, implying a change of radiative energy $\Delta E_{\mathrm{rad}}$; and (iii) an increase of space velocity from the initial value $v_{0}$ to the current value $v$, implying a change of kinetic energy $\Delta E_{\text {kin }}$. These changes are assumed to be connected by $\Delta E_{\mathrm{rad}}+\Delta E_{\mathrm{kin}}=\Delta E_{\mathrm{rot}}$. This means that the radiation loss and increase of kinetic energy are both at the expense of a rotational energy loss. It is shown that this energy conversion occurs during times of order of $10^{-4} \mathrm{~s}$ if the neutron stars are born with magnetic fields in the range of $10^{15}-10^{16} \mathrm{G}$ and initial periods in range $1-20 \mathrm{~ms}$. It is shown that the birth accelerations of neutron stars are of the order of $10^{8} \mathrm{~g}$.
\end{abstract}

Keywords. Neutron stars

\section{Introduction}

The idea that neutron stars are born with magnetic fields typical of magnetars $\left(10^{15}\right.$ $10^{16} \mathrm{G}$ ) and periods typical of millisecond pulsars $(1-20 \mathrm{~ms})$ is based on the assumption that neutron stars experienced three related physical processes occurring at the end of their birth. Because of magneto-rotational instabilities occurring in neutron stars during their birth, these stars could experience an abrupt change of rotational energy which could cause a loss of radiative energy and a gain in kinetic energy:

$$
\Delta E_{\mathrm{rad}}+\Delta E_{\mathrm{kin}}=\Delta E_{\mathrm{rot}} .
$$

A similar energy conversion but with a different radiative term is the basis of the "Rocket Model" proposed by Harrison \& Tademaru (1975). The idea of a loss of rotational energy during the birth process of a neutron star was already considered by Usov (1992) for the case of millisecond pulsars. On the other hand, Spruit (2008) has suggested that a differential rotation in the final stages of the core collapse process can produce magnetic fields typical of magnetars. He has pointed out that some form of magneto-rotational instability may be the cause of an exponential growth of the magnetic field; but that once formed in the core collapse, this magnetic field may decay again through magnetic instabilities. In connection with the idea that neutron stars are born as magnetars, Geppert \& Rheinhardt (2006) have discussed a magneto-hydrodynamical process that significantly reduces the initial magnetic field of a newly-born neutron star in fractions of a second. According to these authors, such a field reduction is due to magneto-hydrodynamical instabilities, which seem to be inevitable if neutron stars are born as magnetars.

The present work focusses on the initial dynamics of neutron stars. Specifically, by considering Eq. (1.1) and the assumption that neutron stars are born with magnetic fields of magnetars and periods of millisecond pulsars, the birth acceleration of neutron stars will be estimated. 


\section{The birth-ultrafast-magnetic-field-decay model of neutron stars}

The Larmor formula for the power radiated by a time-varying magnetic dipole moment $P=2 \ddot{\mu}^{2} /\left(3 c^{3}\right)$, and the estimate $\ddot{\mu} \sim \mu_{0} / \tau^{2}$, where $\tau$ is the characteristic time in the exponential field decay law $B(t)=B_{0} e^{-t / \tau}$, imply the equation $P \simeq 2 \mu_{0}^{2} /\left(3 c^{3} \tau^{4}\right)$, which can be used together with the relation $\mu_{0}=B_{0} R^{3} / 2$ to yield the power radiated by a neutron star of radius $R$ and an initial magnetic field $B_{0}$ :

$$
P \simeq \frac{B_{0}^{2} R^{6}}{6 c^{3} \tau^{4}}
$$

Consider now the specific time $\tau_{s}$ elapsed during the field decay from the initial value $B_{0}$ to the current surface magnetic field $B_{s}$. The condition $B\left(\tau_{s}\right)=B_{s}$ and the law $B(t)=B_{0} e^{-t / \tau}$ imply $B_{s}=B_{0} e^{-\tau_{s} / \tau}$ or equivalently

$$
\tau_{s}=\tau \ln \left(B_{0} / B_{s}\right) .
$$

From Eqs. (2.1) and (2.2) it follows that the change of electromagnetic energy radiated $\Delta E_{\mathrm{rad}} \simeq \tau_{s} P$ is explicitly given by

$$
\Delta E_{\mathrm{rad}} \simeq \frac{B_{0}^{2} R^{6} \ln \left(B_{0} / B_{s}\right)}{6 c^{3} \tau^{3}}
$$

The initial magnetic field $B_{0}$ is associated with the initial angular frequency $\Omega_{0}$ (or equivalently with its associated initial period $P_{0}$ ). The initial rotational kinetic energy of a neutron star is $I \Omega_{0}^{2} / 2$, where $I=2 M R^{2} / 5$ is the moment of inertia for a neutron star with mass $M$. The rotational kinetic energy associated with the surface magnetic field $B_{s}$ is $I \Omega_{s}^{2} / 2$, where $\Omega_{s}$ is the current angular frequency (and $P_{s}$ its associated period) of the neutron star. It is expected that the angular frequency of neutron stars decreases during the field decay of this star. This means that $\Omega_{0}>\Omega_{s}$ and so the rotational kinetic energy must decrease. Since $\Omega=2 \pi / P$ it follows that the period increases during the birth process $\left(P_{s}>P_{0}\right)$. By assuming that the change of radiative energy in Eq. (2.3) and the change of kinetic energy $E_{\text {kin }}=M v^{2} / 2$ of the neutron star (the initial velocity is taken to be zero) are both at the expense of the change of rotational energy $\Delta E_{\text {rot }}=4 \pi^{2} M R^{2}\left(P_{0}^{-2}-P_{s}^{-2}\right) / 5$ the energy conservation reads

$$
\underbrace{\frac{B_{0}^{2} R^{6} \ln \left(B_{0} / B_{s}\right)}{6 c^{3} \tau^{3}}}_{\Delta E_{\mathrm{rad}}}+\underbrace{\frac{M v^{2}}{2}}_{\Delta E_{\mathrm{kin}}}=\underbrace{\frac{4 \pi^{2} M R^{2}}{5}\left(\frac{1}{P_{0}^{2}}-\frac{1}{P_{s}^{2}}\right)}_{\Delta E_{\mathrm{rot}}} .
$$

To get an idea of the order of the time $\tau$, consider the Crab pulsar B0531+21 which has $M=1.4 M_{\odot} ; R=10 \mathrm{~km} ; P_{s}=0.033 \mathrm{~s} ; B_{s}=3.78 \times 10^{12} \mathrm{G} ; v_{\perp}=141 \mathrm{~km} / \mathrm{s}$ which implies $v=172.7 \mathrm{~km} / \mathrm{s}$, where the relation $v \approx \sqrt{3 / 2} v_{\perp}$ has been used (Lyne \& Lorimer 2006). It has been suggested that the Crab pulsar was born with $P_{0} \approx 0.019 \mathrm{~s}$ (Lyne et al. 1993). Using this initial period and assuming $B_{0}=5.8 \times 10^{15} \mathrm{G}$, Eq. (2.4) yields $\tau \approx R / c$. For the magnetar J1809-1943, Eq. (2.4) also implies $\tau \approx R / c$ if $P_{0} \approx 0.02 \mathrm{~s}$ and $B_{0}=9.5 \times 10^{15} \mathrm{G}$. For the isolated millisecond pulsar MSP B1257+12, Eq. (2.4) yields $\tau \approx R / c$ if $P_{0} \approx 0.059 \mathrm{~s}$ and $B_{0}=2.7 \times 10^{15} \mathrm{G}$. The time $\tau=R / c$ agrees with the idea that neutron stars are born with magnetic fields in the range of $10^{15}-10^{16} \mathrm{G}$ and initial periods in range of 1-20 ms. Magnetic field decays from one to eight orders of magnitude satisfy $2.3 \leqslant \ln \left(B_{0} / B_{s}\right) \leqslant 4.4$. When this relation and $\tau=R / c$ are used in Eq. (2.2) one has

$$
\tau_{s} \sim 10^{-4} \mathrm{~s} \text {. }
$$

This means that the field decay from $B_{0}$ to $B_{s}$ is ultrafast if $B_{0}$ lies in the range of $10^{15}-10^{16} \mathrm{G}$ and $P_{0}$ in the range of $1-20 \mathrm{~ms}$. 


\section{Birth accelerations of neutron stars}

The energy conversion in Eq. (2.4) occurs suddenly and therefore one can write

$$
v \approx a \tau_{s}
$$

where $a$ is the birth acceleration. Equations (2.2), (2.4), (3.1) and $\tau=R / c$ yield

$$
a=\sqrt{\frac{8 \pi^{2} c^{2}\left(P_{0}^{-2}-P_{s}^{-2}\right)}{5 \ln \left(B_{0} / B_{s}\right)^{2}}-\frac{B_{0}^{2} R c^{2}}{3 M \ln \left(B_{0} / B_{s}\right)}} .
$$

Using this equation with $M=1.4 M_{\odot}$ and $R=10 \mathrm{~km}$, and taking the specific values for $B_{s}$ and $P_{0}$ from the ATNF Pulsar Catalogue and assuming values for $P_{0}$ and $B_{0}$, the birth acceleration can be calculated and expressed in terms of $\mathrm{g}=9.8 \mathrm{~m} / \mathrm{s}^{2}$. Four sets of neutron stars with reported transverse velocities will be now considered:

Interval: $2 \mathrm{~s}<P_{s} \leqslant 8.5 \mathrm{~s}$.

There are 9 neutron stars in this interval. The average values are $P_{s}=4.82 \mathrm{~s}$ and $B_{s}=3.14 \times 10^{13} \mathrm{G}$. If the values $P_{0}=0.02 \mathrm{~s}$ and $B_{0}=7 \times 10^{15} \mathrm{G}$ are assumed then Eq. (3.2) predicts the birth acceleration $a=5.0 \times 10^{8} \mathrm{~g}$.

Interval: $1 \mathrm{~s} \leqslant P_{s} \leqslant 2 \mathrm{~s}$.

There are 29 neutron stars in this interval. The average values are $P_{s}=1.32 \mathrm{~s}, B_{s}=$ $4.17 \times 10^{12} \mathrm{G}$. If the values $P_{0}=0.02 \mathrm{~s}$ and $B_{0}=5 \times 10^{15} \mathrm{G}$ are assumed then Eq. (3.2) gives the birth acceleration $a=5.8 \times 10^{8} \mathrm{~g}$.

Interval: $0.02 \mathrm{~s} \leqslant P_{s}<1 \mathrm{~s}$.

There are 130 neutron stars in this interval. The average values are $P_{s}=0.41 \mathrm{~s}$ and $B_{s}=1.28 \times 10^{12} \mathrm{G}$. If the values $P_{0}=0.02 \mathrm{~s}$ and $B_{0}=5 \times 10^{15} \mathrm{G}$ are assumed then Eq. (3.2) implies the birth acceleration $a=4.5 \times 10^{8} \mathrm{~g}$.

Interval: $0.0015 \mathrm{~s} \leqslant P_{s}<0.009 \mathrm{~s}$.

There are 9 millisecond pulsars in this interval. The average values are $P_{s}=0.005 \mathrm{~s}$ and $B_{s}=2.59 \times 10^{8} \mathrm{G}$. If the values $P_{0}=0.0049 \mathrm{~s}$ and $B_{0}=10^{15} \mathrm{G}$ then Eq. (3.2) predicts the birth acceleration $a=3.1 \times 10^{8} \mathrm{~g}$.

It can be concluded that birth accelerations of neutron stars occur in times $\tau_{s} \sim 10^{-4} \mathrm{~s}$ and are of the order of $10^{8} \mathrm{~g}$. If for example, $a=5 \times 10^{8} \mathrm{~g}$ and $\tau_{s} \sim 10^{-4} \mathrm{~s}$ are assumed then the birth velocity (kick velocity) is of the order of $500 \mathrm{~km} / \mathrm{s}$. This value is approximately the average value for pulsar velocities found by Lyne \& Lorimer (2006).

\section{References}

Geppert, U. \& Rheinhardt, M. 2006, $A \& A, 456,639$

Harrison, E. R. \& Tademaru, E., 1975, ApJ, 201, 447

Lyne, A., et al., 1993, MNRAS, 265, 1003

Lyne, A. \& Lorimer D., 2006, Nature, 369, 127

Spruit, H. C., 2008 in Cosmic Magnetic Fields: From Planets, to Stars and Galaxies. Eds. K. G. Strassmeier, A. G. Kosovichev and J. E. Beckman. IAU Symposium, 259, 61

Usov, V. V., 1992, Nature, 357, 472 